\title{
Whole body diffusion-weighted MRI in detection of metastasis and lymphoma: a prospective longitudinal clinical study
}

\author{
Heba H. Goda ${ }^{1 *} \mathbb{D}$, Abd Elkareem H. AbdAllah', Eman A. Ahmed ${ }^{1}$, Hassan I. Megally', Marwa I. Khalaf²,
} Ahmed M. Taha ${ }^{3}$ and Hosam Eldeen G. Mohamed ${ }^{1}$

\begin{abstract}
Background: Whole-body diffusion-weighted magnetic resonance imaging (WB-DWI-MRI) is an emerging tool that has an increasing role in the diagnosis of metastasis and lymphoma. This is a longitudinal study in actual clinical settings designed to assess WB-DWI-MRI in detection of tumor spread. The study included all patients who were referred to Radiology Department, during the period from June 2016 till May 2018, with either a known primary tumor (either laboratory, radiologically, or histologically proven, of any type, affecting any organ) or with biopsyproven lymphoma of any subtype, affecting any organ. All patients underwent WB coronal T1-weighted, STIR, axial T2-weighted, and DWI-MRI examinations before commencing any treatment with curative intent. The body was divided into lymph nodes (LNs), skeletal system, and organs (brain, lung, and liver). Patients were followed up till the nature of the lesion(s) was confirmed (clinically, radiologically, or histologically).
\end{abstract}

Results: The study included 46 patients; 27 patients had metastases and 19 had lymphomas. Sensitivities, specificities, and accuracies for LN detection were $77 \%, 85 \%$, and $83 \%$; for skeletal metastasis were $88 \%, 94 \%$, and 92\%; for brain lesions were 78\%, 95\%, and 91\%; and for lung lesion were 64\%, 88\%, and 76\%, respectively. As for the liver, all lesions were correctly identified and did not miss any lesion with accuracy of $100 \%$. Overall, 1739 lesions were discovered in 1271 regions out of 3818 examined regions with overall sensitivity, specificity, and accuracy of $86 \%, 92 \%$, and $90 \%$ respectively.

Conclusion: The diagnostic performance of WB-DWI-MRI is variable among different anatomical sites. It has good performance in diagnosis of some organs as liver, bone marrow, and some LNs regions as porta-hepatis. It has a less diagnostic performance in the lung, and LNs located in cervical, mediastinum, supraclavicular, and mesenteric regions.

Keywords: WB-MR imaging, DWI, Metastasis, Lymphoma, Neoplasm

\section{Background}

Metastatic lesions are common manifestations of advanced cancers with very high prevalence in current practice. It is becoming an everyday clinical question [1]. Malignant lymphoma is another relatively common tumor that requires accurate staging at initial presentation that is essential for treatment planning and

\footnotetext{
* Correspondence: hebagoda08@googlemail.com

${ }^{1}$ Radiology Department, Faculty of Medicine, Assiut University, Assiut, Egypt Full list of author information is available at the end of the article
}

prognostication. Clinicians are highly dependent upon different imaging procedures for staging that takes into account the number of involved sites and the type of lesions (nodal or extra-nodal) [2].

Whole-body diffusion-weighted magnetic resonance imaging (WB-DWI-MRI) is an emerging tool that has been developed to assess tumor spread. Combining MRI's unique power of characterizing tissues, based on water and fat content, with DWI's ability to assess tissue water motion, provides a unique tool to both detect and 
characterize lesions within tissues. Positron emission/ computed tomography (PET/CT) is considered the preferred investigation in secondaries detection in clinical guidelines [3]. WB-DWI-MRI is probably the closest competitor to PET/CT scanning. As PET/CT, it covers the whole body and gives exquisite anatomical identification as it is always done in association with other MRI sequences and has the capability to be considered "functional" scanning. But unlike PET/CT, it avoids ionizing radiation, of lower cost, and does not require radioactive tracers. Also, PET/CT has low sensitivity in detecting metastasis in the brain and liver, due to the high physiological tracer uptake in these organs [3].

In this study, we tried to explore the value of WBDWI-MRI in detecting metastases, in clinical settings, by including different types of malignancies and lymphomas in a single study.

\section{Methods}

The study was prospective. Written informed consents were obtained from all patients for their data to be included in the study. Institutional ethical committee approval was obtained. The study included all patients referred to Radiology Department, during the period from June 2016 till May 2018, with a known primary or lymphoma, that were laboratory, radiologically, or histologically proven, of any type, affecting any organ. All patients underwent WB-DWI-MRI before commencing any treatment of the primary lesion (surgery, radiotherapy or chemotherapy). All included patients had no contraindication for MRI examination. The study excluded patients in whom a confirmatory method of the nature of the possibly metastatic lesion was not available. Patients who did not show any metastatic lesion in the scans were also excluded.

\section{Confirmation of the nature of the lesion}

The identified lesion(s) was considered a metastasis if it was confirmed histologically by a diagnostic biopsy that was done for clinical needs. Alternatively, if this was not available, clinical and radiological progress were followed till identification of the true nature of the lesion. All radiological investigations done during the follow-up period were requested for the clinical needs of patients, and at the discretion of the responsible physician.

\section{MRI technique}

All patients had MRI according to the study protocol. The machine used was Gyroscan Philips 1.5 Tesla strength ACS-NT synergy coil, Holland. No contrast media was used. The patients lied supine on MRI table with hands beside his body. Conventional techniques used were coronal T1-weighted images (WI), coronal STIR, axial T2-WI images, and sagittal STIR of the spine. Then, WB-DWI-MRI was obtained. Parameters are summarized in Table 1. Images were acquired with a built-in body coil for signal reception, from head to toes, under free breathing. The total acquisition time for WBconventional and DWI-MRI was approximately 40-45 min including 20-25 min for WB-DWI. Axial images were reconstructed on a coronal plane; then the reconstructed images in the coronal plane for each station were merged to obtain a coronal WB images. Colorcoded fused T1-DWI images and apparent diffusion coefficient (ADC) maps were generated by Phillips workstation software.

\section{Image interpretation}

The images for MRI were prospectively assessed for each patient by two radiologists who have more than 5 years' experience in MRI imaging. For the purpose of the research, the body was divided into three groups: lymph nodes (LNs), skeletal system, and organs. Then, LN groups were further divided into 16 regions and skeletal system was divided into 63 regions. Organs considered in the study are the three organs commonly involved which are the brain, lungs, and liver, and a fourth group that includes the other organs. Criteria for involvement were either local or diffuse hypo intensities in T1-WI or hyper intensities in T2-WI and STIR images or restricted diffusion in DWI or hypo-intensity on ADC maps or their combinations. Post-processing ADC values and maps were done separately for each anatomical location through the workstation software. Lowest ADC values were measured using a region of interest in the center of the lesion avoiding necrotic and cystic areas. A cut-off value of $1 \times 10^{-3} \mathrm{~mm}^{2} / \mathrm{s}$ was used to differentiate restricted areas from non-restricted areas. A LN was considered positive if its short diameter was equal to or more than $10 \mathrm{~mm}$, loss of fatty hilum, irregular margins or apparent speculation, diffusion restriction, and low ADC value where a cut-off value of $0.8 \times 10^{-3} \mathrm{~mm}^{2} / \mathrm{s}$ was used.

\section{Statistical analysis}

Analysis will be performed using SPSS 18.0 software (SPSS Chicago, IL, USA). The sensitivity, specificity, accuracy, positive predictive value, and negative predictive value were calculated and expressed per region not per patient.

\section{Results}

This study included 46 patients; $(25$ males and 21 females, median age was 49 , range 16 to 79 years); 27 patients had metastases of a known primary tumor and 19 had lymphomas. Distribution according to primary site is shown in Table 2. 
Table 1 Parameters of WB-MRI technique

\begin{tabular}{lllll}
\hline & T1W & T2W & STIR & DWI $(b$ value 0,1000) \\
\hline TR; ms & 537 & 1179 & 6564 Inversion time 130 & 6960 Inversion time 150 \\
TE; ms & 20 & 80 & 70 & 79 \\
Reconstruction matrix & $512 \times 512$ & $512 \times 512$ & $512 \times 512$ & $512 \times 512$ \\
Slice thickness; mm & 7 & 7 & 7 & 7 \\
\hline
\end{tabular}

\section{Methods of confirmation of the nature of the lesions}

Radiological follow-up by different imaging techniques was the method of confirmation of the nature of the lesion in most of the occasions. Follow-up ranged from 6 to 12 months. Table 3 shows the number of scans done in patients in this study that were used for confirmation. Most patients had underdone more than one imaging modality during follow-up. Only 4 lesions had been biopsied; 2 from liver, 1 from lung, and 1 from a neck LN.

\section{Diagnostic performance of WB-MRI}

Results are shown in Tables 4 and 5. Regarding LN detection, WB-DWI-MRI detected 233 out of all 736 possible LN regions in all patients. Sensitivity, specificity, and accuracy for LN detection were $77 \%, 85 \%$, and $83 \%$, respectively. WB-DWI-MRI showed highest sensitivity in femoral groups of LNs followed by inguinal and porta-hepatis groups of LN's. On the other hand, it was the lowest in the supraclavicular and mediastinal groups of LNs. The highest false-positive results were in axillary and inguinal LNs, while the mediastinal, supraclavicular, and cervical LNs showed the highest false-negative results (Fig. 1).

For skeletal metastasis, WB-DWI-MRI detected lesions in 984 out of 2898 regions in all patients. Sensitivity, specificity, and accuracy were $88 \%, 94 \%$, and $92 \%$, respectively. In all regions, sensitivity ranged between 80 and $92 \%$ except for pubic bones where it was 60\% (Fig. 2).

Table 2 Distribution according to primary site

\begin{tabular}{ll}
\hline Organ & Frequency \\
\hline Breast & 8 \\
Prostate & 5 \\
Liver & 4 \\
Colon & 3 \\
Thyroid & 2 \\
Lung & 2 \\
Ovaries & 2 \\
Testes & 1 \\
HL & 11 \\
NHL & 8 \\
Total & 46 \\
\hline
\end{tabular}

Abbreviations: HL Hodgkin's lymphoma, NHL non-Hodgkin's lymphoma
For brain lesions, sensitivity, specificity, and accuracy were $78 \%, 95 \%$, and $91 \%$, respectively. In 46 patients, 9 patients had 13 brain lesions; of them, 2 were falsepositive and 2 were false-negative. For lung lesions, sensitivity, specificity, and accuracy were $64 \%, 88 \%$, and $76 \%$ respectively. Fifty-four lesions were identified in 17 patients; of them, 3 were false-positive and 8 were falsenegative (Fig. 3 and 4). As regards the liver, WB-DWIMRI detected correctly all 52 lesions in 16 patients and did not miss any lesion with accuracy of $100 \%$ in this study (Fig. 5).

Overall, 1739 lesions were discovered by WB-DWI-MRI in 1271 regions out of 3818 examined regions; 1069 regions were true-positive and 176 regions were missed and were later proved to be malignant, whereas 202 regions were false-positive. Overall sensitivity, specificity, and accuracy were $86 \%, 92 \%$, and $90 \%$, respectively.

\section{Discussion}

This research was intended to be an exploratory research of the applicability of WB-DWI-MRI. It was a prospective clinical study, in which the patients were followed up till a clarification of the nature of the lesion had been reached. So, the researchers are naturally blind to the actual nature of the lesion. In most similar studies, comparisons are made with other concurrently done scans that have their own inherent accuracies. Additionally, for this study to be as beneficial and reproducible as possible, we tried to simulate the actual clinical scenario that the radiographer is exposed to in his/her daily activities. So, we combined the data from the conventional MRI to that of DWI-MRI and did not try to compare

Table 3 Scans done for confirmation of the nature of the lesions

\begin{tabular}{ll}
\hline Mode of confirmation & Number of patients \\
\hline CT Chest & 31 \\
Ultrasound abdomen and pelvis & 31 \\
CT/MRI abdomen and pelvis & 29 \\
Bone scan & 22 \\
Ultrasound neck & 13 \\
PET/CT & 12 \\
Ultrasound/mammography/MRI breast & 8 \\
Biopsy & 4 \\
MRI FLAIR for brain lesions & 2 \\
\hline
\end{tabular}




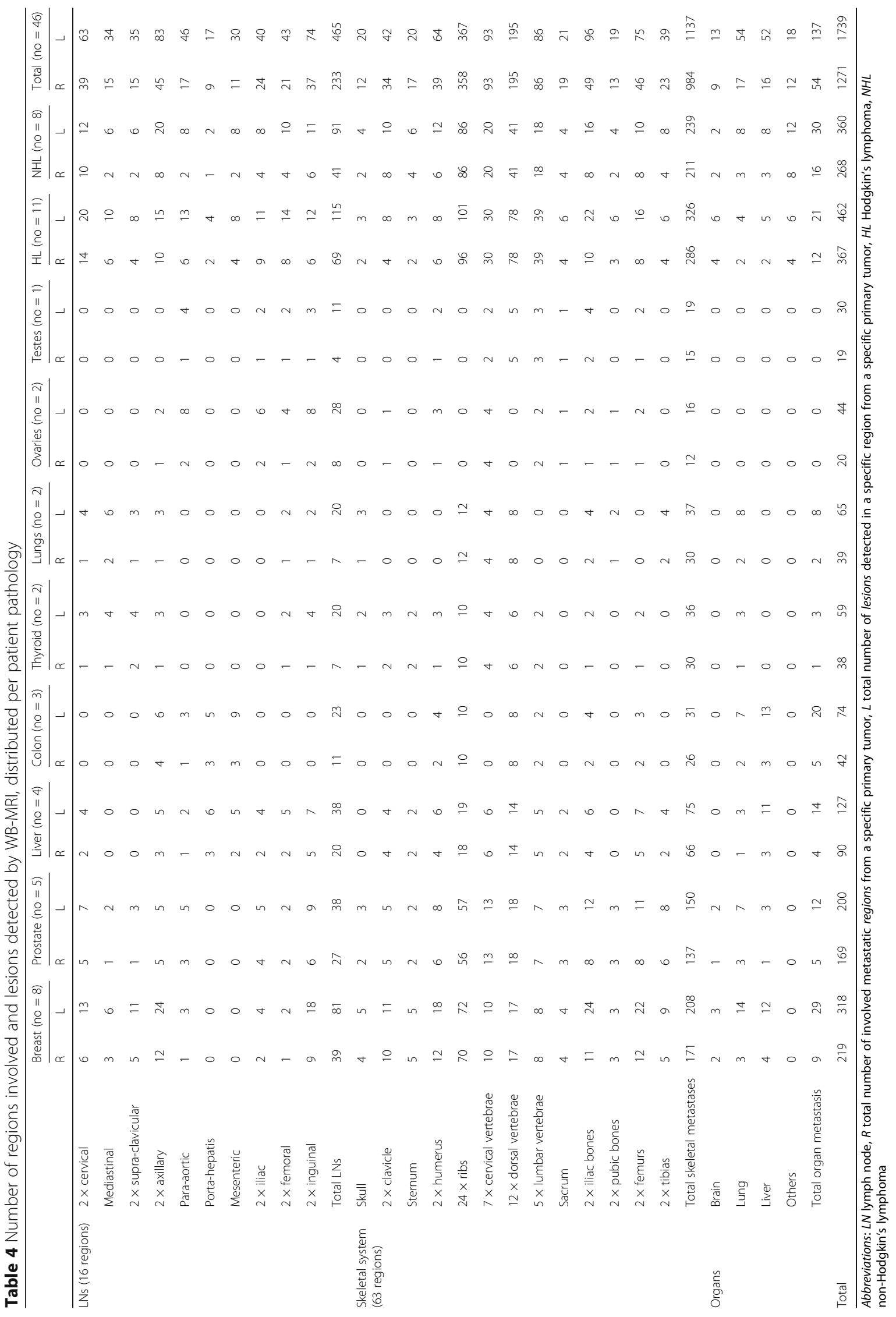




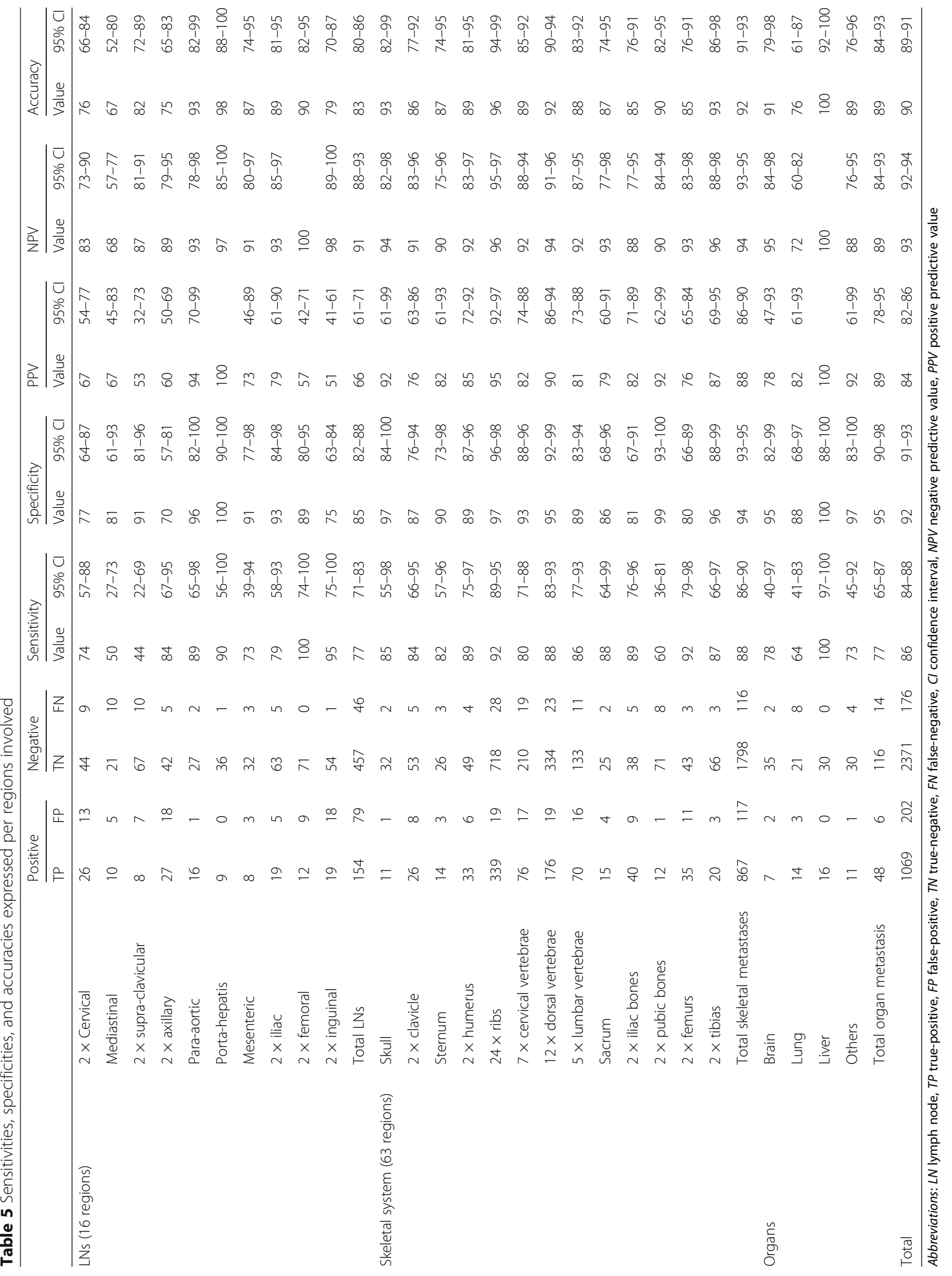




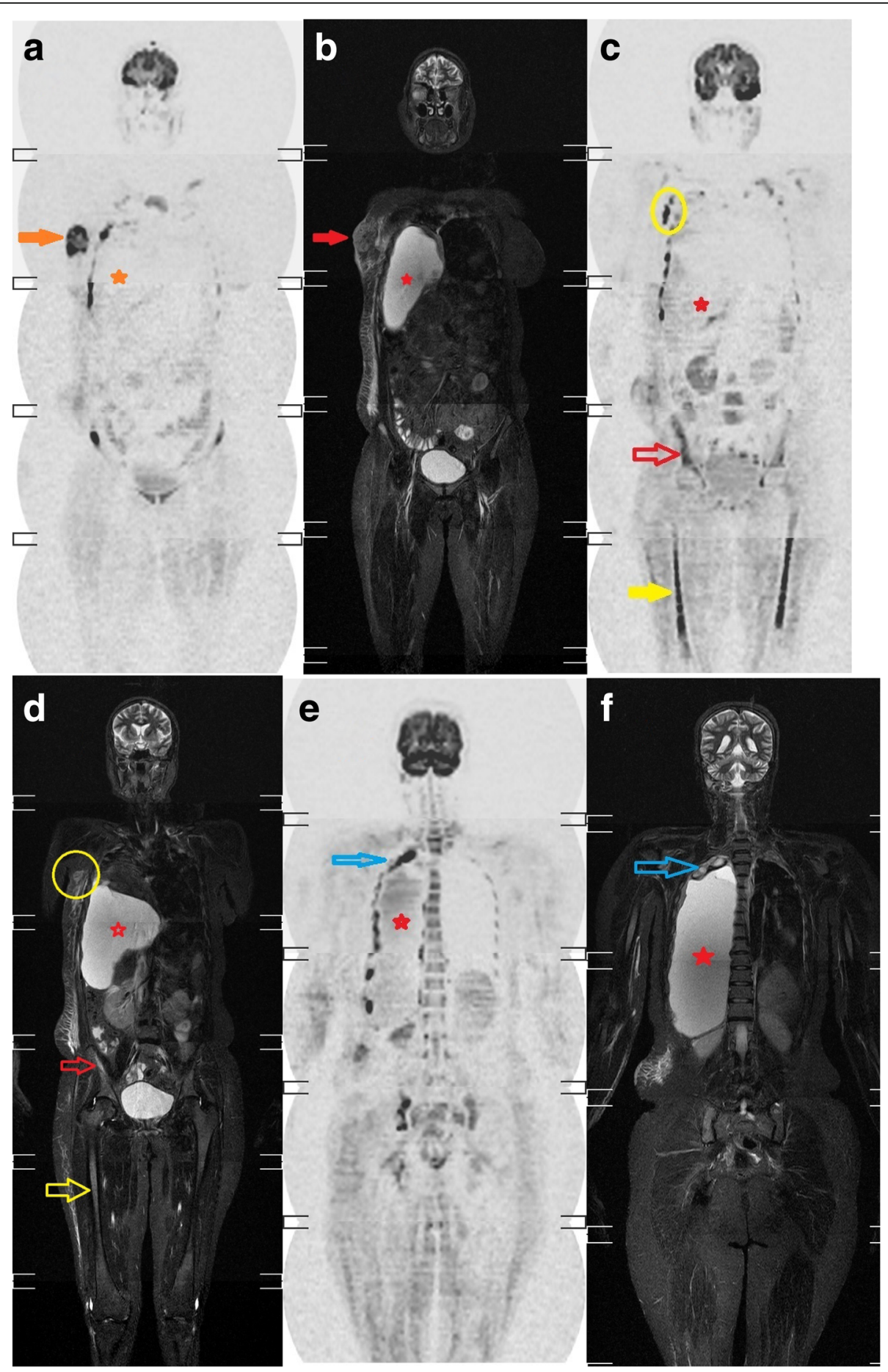

Fig. 1 A 54-year-old female patient diagnosed with metastatic breast cancer. a Inverted grey scale coronal WB-DWI and $\mathbf{b}$ coronal STIR show the primary right breast cancer (red arrows). $\mathbf{c}$ Inverted grey scale coronal WB-DWI and $\mathbf{d}$ coronal STIR show metastatic right axillary lymph nodes; the transverse diameter of one of them is $1.3 \mathrm{~cm}$ (yellow circle), metastasis of both femurs (yellow arrow) and right iliac bone (red arrow) shown as hyperintensity in STIR and restricted diffusion in DWI. e Inverted grey scale coronal WB-DWI and $\mathbf{f}$ coronal STIR show metastatic first right two ribs as well as multiple rib lesions (blue arrows). Marked right pleural effusion is noted in coronal STIR but does not show restricted diffusion in DWI (red stars) 


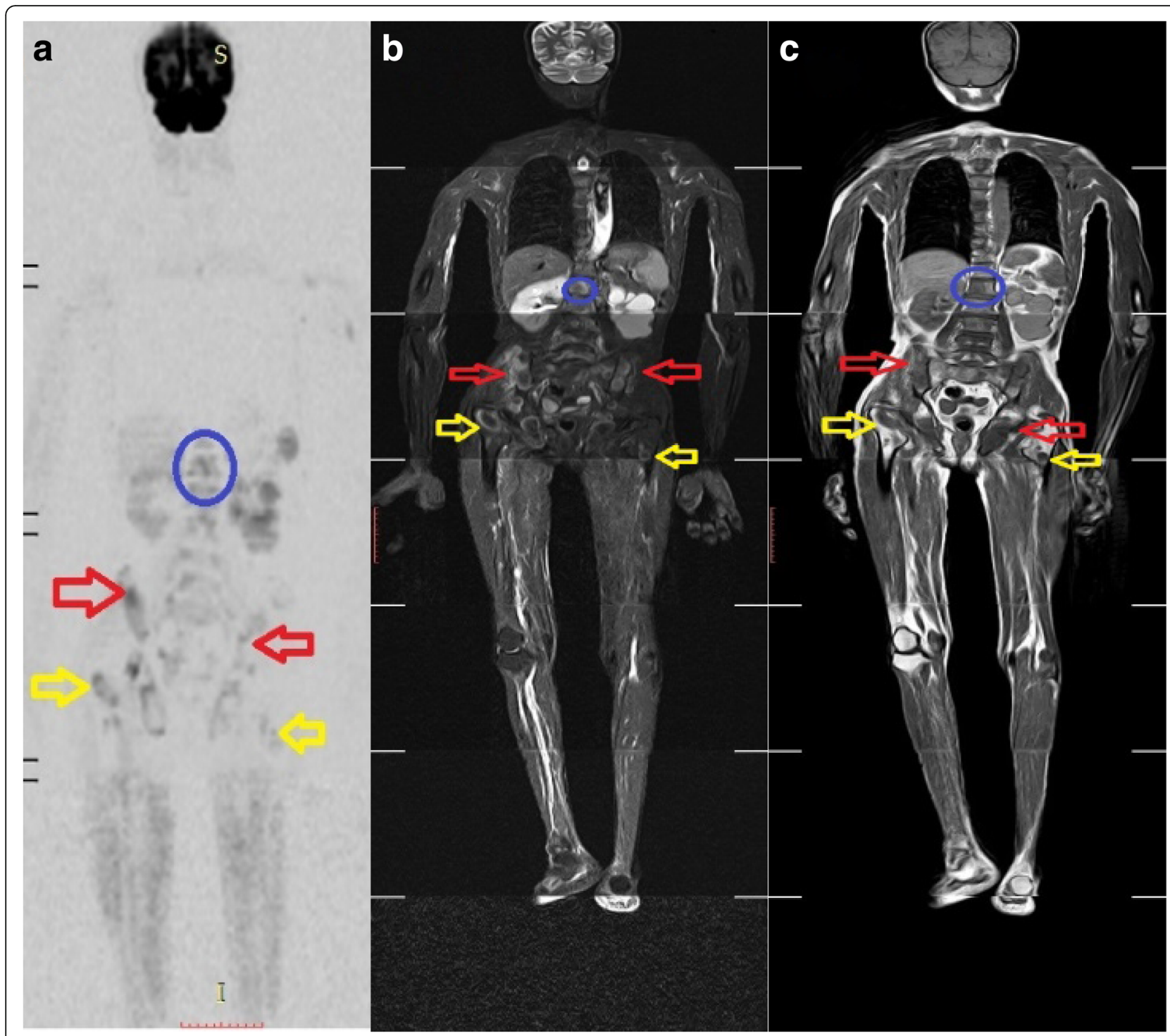

Fig. 2 A 62-year-old male patient with cancer prostate. Multiple bone metastases in vertebral bodies (blue circle), bilateral iliac bones (red arrows), and bilateral femoral heads (yellow arrows). a Inverted gray scale coronal WB DWI. b Coronal STIR. c Coronal T1 WI

them to each other, which does not happen in the clinical scenarios.

We did not restrict our inclusion to a specific disease or organ, either primary or secondary. Also, to get the highest yield from our limited research, we deliberately did not investigate the role of WB-MRI in assessment of tumor response.

In this study, to get the most accurate calculations, we divided the LN and skeletal groups to the smallest possible segments or regions. For example, ribs, we divided in 24 ribs; so every single rib is an independent region. Meanwhile, we did not divide organs into further smaller segments.

\section{LN lesions}

Regarding evaluating malignant metastatic or lymphomatous LNs, the relatively low sensitivity of WB-DWIMRI in detection of regional nodal metastases in this study can be explained by the fact that metastases can be present in non-enlarged LN and not all enlarged nodes are malignant. Changing the size criteria towards larger or smaller cut-offs will influence sensitivity and specificity as a low threshold for cut-off values would increase the sensitivity, but specificity would decrease. This can explain the variation in the sensitivity of WBDWI-MRI in other studies which ranges from 60 to $90 \%$ $[4,5]$. The most accepted criterion for malignant $\mathrm{LN}$ 


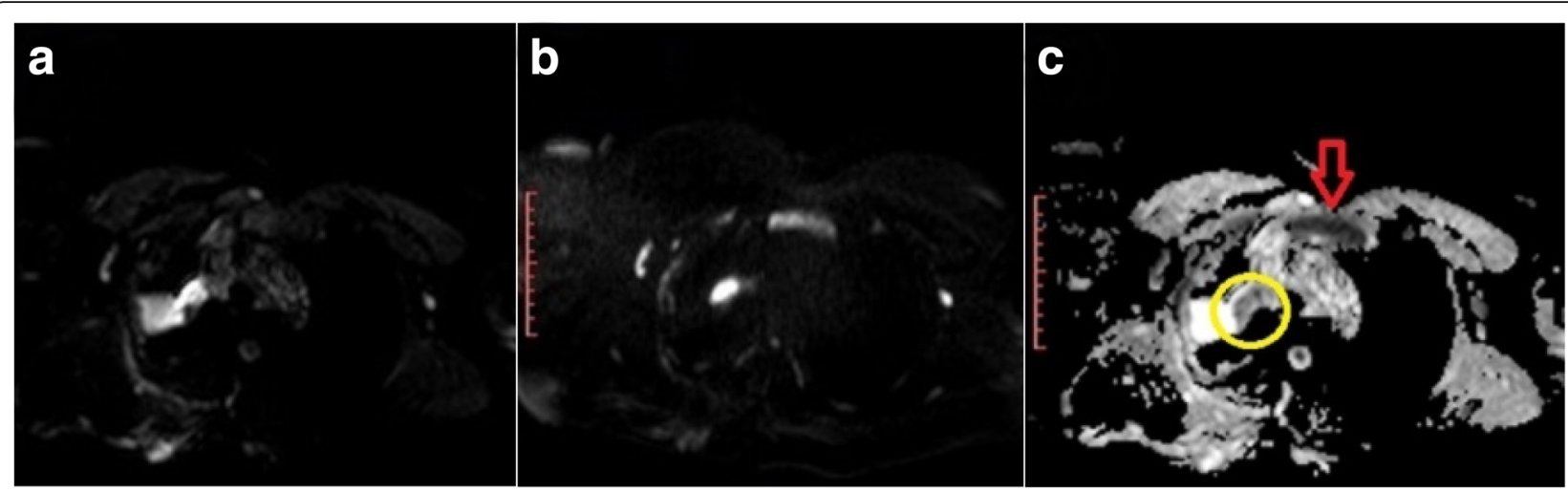

Fig. 3 A 45-year-old female patient with right apical lung cancer, epsilateral pleural effusion, and sternal metastasis. a Axial DWI. Medially located tumor and laterally located pleural effusion; both are showing restricted diffusion. b Axial DWI. Restricted diffusion of sternal metastasis. $\mathbf{c}$ ADC map. Low ADC in malignant tumor (yellow circle) and sternal metastasis (red arrow) but high in pleural effusion

involvement in conventional MRI sequences is that a short diameter greater than $8-10 \mathrm{~mm}$ [6]. A normal LN already has a relatively long $\mathrm{T} 2$ relaxation time and a restricted diffusion, due to their high cellularity, thus adding ADC values enhanced the discrimination between malignant and benign LNs. In this study, the combined usage of WB-DWI-MRI with the conventional sequences improved the detectability of LN metastasis. Seber et al. [7] in their study on differentiating benign from malignant LN infiltration from wide range of tumor types involving different body locations, found that adding DWI (utilizing an ADC cut-off value of $0.8 \times 10^{-3} \mathrm{~mm}^{2} / \mathrm{s}$ ) to conventional MRI findings increased the accuracy from 80.6 to $91.6 \%$.

There is a wide variability of accuracies within different sites in the study. The highest accuracy was found in porta-hepatis LNs. Whereas the lowest accuracy was found in mediastinal LNs where image quality may be affected by pulsation artifacts. The later finding was contrary to Sigovan et al.'s findings [8] where they showed high sensitivity, specificity, and accuracy (90.9\%, 83\%, and $85 \%$, respectively) for DWI in differentiating benign from malignant enlarged mediastinal LNs.

One of the confusing pitfalls is splenic hilar lymphadenopathy with an accessory spleen, as both may be hyper-intense on STIR and DWI sequences. The same issue was reported by Massani et al. [9].

In our study, there were no differences in DWI values between Hodgkin disease and non-Hodgkin's lymphoma. Interestingly, Sabri et al. [10], in their study on malignant mediastinal LN involvement, detected a significant difference between the ADC values of Hodgkin disease and non-Hodgkin lymphoma.

\section{Skeletal system lesions}

In this study, WB-DWI-MRI identified 1137 bony lesions in 984 regions. The sensitivity, specificity, and accuracy were $88 \%, 94 \%$, and $92 \%$, respectively. Similar to other studies, we noted that the sole evaluation of bone lesions by DWI, STIR, or T2-WI makes high falsepositive results of non-tumor lesions as in anemia, smoking, spondylodiscitis, osteomyelitis, bone infarctions, hemangiomas, and fractures which cause increased signal due to bone marrow edema [11-13]. The use of ADC maps and T1-WI in these occasions could decrease the false-positive results. Sagittal STIR of whole spine was included for evaluation of all the vertebrae to improve the accuracy of lesion localizations due to a higher spatial resolution [14]. False-negative results were found in the areas prone to motion artifacts as the sternum and clavicles. They were more obvious in conventional sequences than in WB-DWI $[15,16]$.

\section{Brain lesions}

WB-DWI-MRI only provide information about tumor cellularity, and this may explain its low sensitivity of $78 \%$ and high specificity $95 \%$ in this study as it could correctly identify 13 lesions of the brain in 9 patients. This was evident in other studies as well and is believed to be due to inherent high signal of the brain [17]. In suspected brain lesions, FLAIR sequence was exceptionally added to confirm the diagnosis, which is capable to elucidate areas of vasogenic edema around the tumor [18]. Ahmed and Mokhtar [19] reported similar finding to ours in their study on cerebral lesions. They found that there are mixed diffusion changes in most of the lesions in their study, and there was overlap in the measured ADC values between neoplastic and nonneoplastic lesions. On the other hand, Berghoff et al. [20] reported that ADC values correlated with survival and recurrence after surgical resection. Additionally, Lee et al. [21] reported that it correlated with survival after radiosurgery. Another interesting finding was reported 


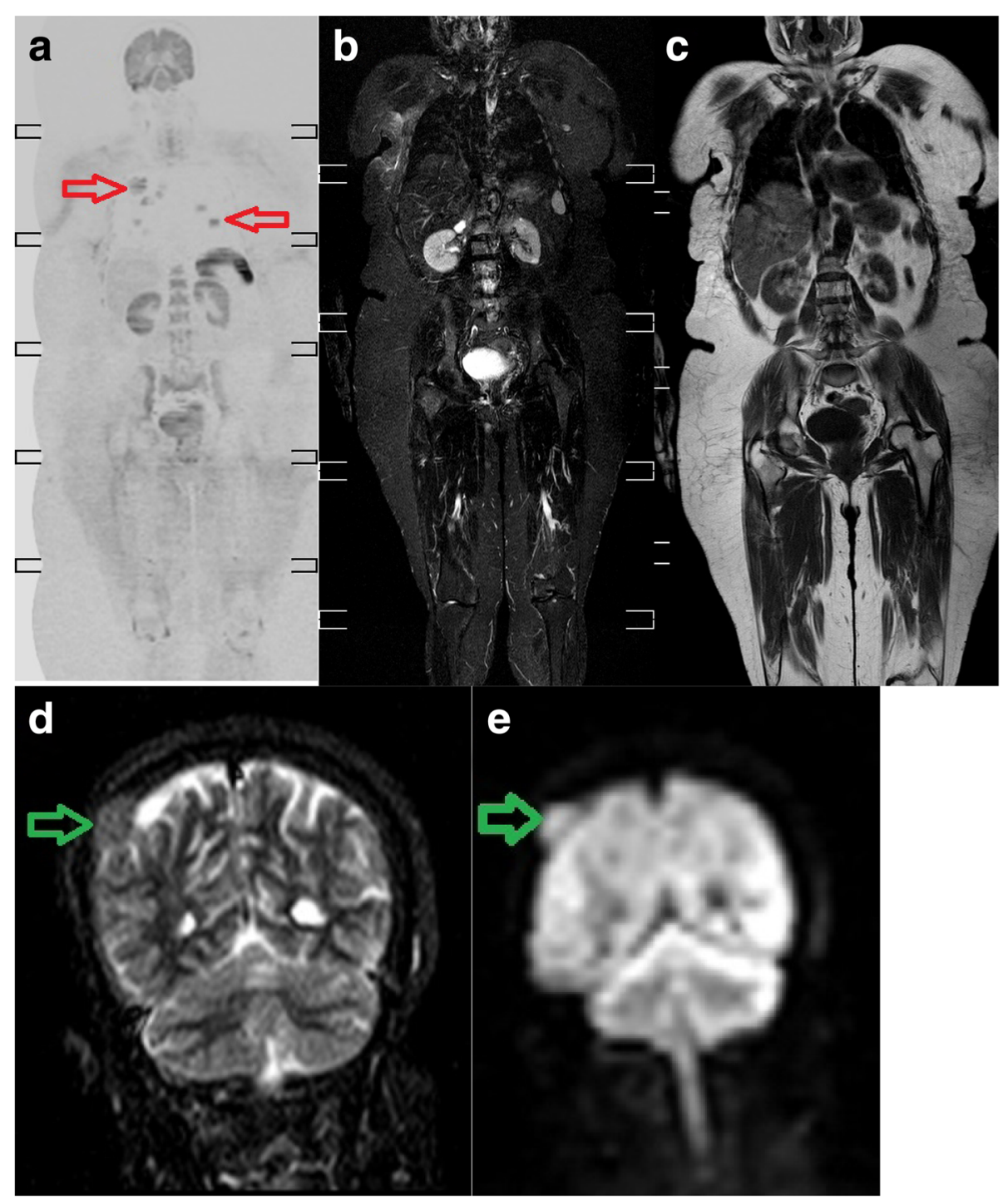

Fig. 4 A 61-year-old female patient, diagnosed with right breast cancer. Metastatic lesions were suspected. a Inverted grey scale coronal WB-DWI: multiple cannon ball metastatic lesions (red arrows) distributed bilaterally which are not seen in coronal STIR (b) or coronal T1 WI (c). Right parietal bone metastatic lesion (green arrows) seen as hyper intensity in coronal STIR (d) and restricted diffusion in coronal DWI (e)

by Zakaria et al. [22], that ADC changes at the tumor edge may indicate a more aggressive phenotype.

\section{Pulmonary lesions}

WB-MRI identified 54 lesions in 17 patients. Sensitivity, specificity, and accuracy were $64 \%, 88 \%$, and $76 \%$, respectively. This was lower than that of other conventional imaging as CT in many studies [23]. This can be attributed to difficulties in detection of small metastatic lung nodules (less than $8 \mathrm{~mm}$ ). Free-breathing DWI acquisition in this study led to reduced accuracy of lesion detection (especially lung bases) due to respiratory movements and cardiac motion [23]. Also, lesions located at air-tissue interfaces were more difficult to be identified. Similarly, Regier et al. [24] reported sensitivity of $97 \%$ for nodules larger than $10 \mathrm{~mm}$, but for $6-9 \mathrm{~mm}$ nodules, the sensitivity decreased to $86 \%$, and for nodules $5 \mathrm{~mm}$ or smaller, it decreased to $43.8 \%$. Similar finding was reported by Liu et al. [25] in assessing pulmonary metastases in patients with renal cell carcinoma. The use of ADC mapping added a value of discrimination between benign and malignant nodules [26]. A recent meta-analysis reported sensitivity and specificity of DWI to be $82.8 \%$ and $80.1 \%$, respectively [27].

\section{Liver lesions}

Regarding hepatic metastases, WB-MRI had an accuracy of $100 \%$. Thanks to the use of combined DWI with ADC together with anatomical localization by conventional MRI, the detection rate of malignant focal hepatic lesion 


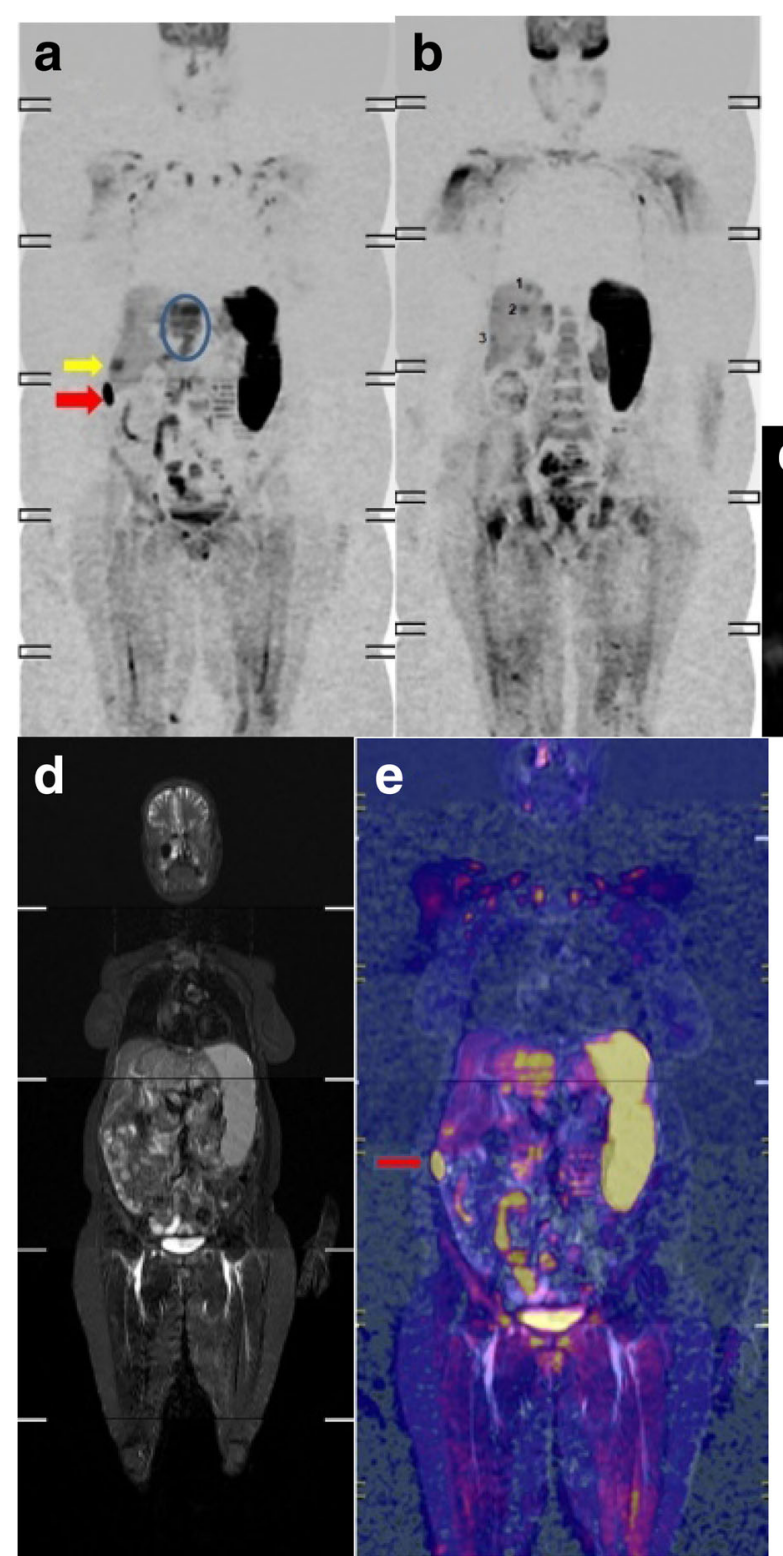

Fig. 5 A 55-year-old female patient with metastatic hepatocellular carcinoma (HCC). a Coronal WB DWI. Left lobe HCC (circle) and a metastatic hepatic focal lesion (yellow arrow). Note splenomegaly. b Coronal WB DWI. Multiple metastatic hepatic focal lesions (three lesions). c Axial DWI. Left lobe HCC and hepatic focal lesions. d Coronal STIR. Liver lesions are not clearly shown as in DWI. e Fusion images of both DWI and STIR. Hepatic flexure of the colon can be misinterpreted as a hepatic focal lesion (red arrow)

was high. These results were also reported in another study [28]. Another explanation to these high results in this study as whole organ is considered a single region. As there was usually more than one lesion in the liver, if only one of them was correctly detected, the researchers would consider this scan a true-positive; the others will not be reported. WB-MRI is comparable with contrastenhanced CT or contrast-enhanced MRI but with advantage that it does not require contrast medium [29]. Of particular interest, Abou-Khadrah and Bedeer [30] in a prospective study showed that DWI is beneficial in small hepatocellular carcinomas smaller than $2 \mathrm{~cm}$ where they reported high sensitivity, specificity, and accuracy of combined conventional MRI and diffusion (92.8\%, 71.4\%, and 93.3\%, respectively).

\section{Study limitations}

There are some limitations to the study. It included a heterogenous group of patients with different pathologies, so, each pathology was represented by a small number of patients. Our aim was to do an exploratory study for the value of WB-DWI-MRI in detecting metastases, 
by including different types of malignancies and lymphomas in a single study. To minimize the effect of small study size, we deliberately did not investigate the role of WB-MRI in assessment of tumor response. Another limitation to the study is that histological examination to confirm the nature of the lesion was possible in only a small number of lesions. This study was conducted in clinical setting, and biopsies were requested by the treating physicians for the clinical requirements not for the sake of the study. This study was prospectively conducted and included only those patients who could be followed up by different radiological examinations for periods that were deemed sufficient to prove that a specific radiological finding is metastatic or not base upon its biological behavior.

\section{Conclusion}

The diagnostic performance of WB-DWI-MRI combined with conventional MRI and ADC mapping is variable among different anatomical sites. It has good performance in diagnosis of some organs as liver, bone marrow, and some LNs as porta-hepatis. It has a less diagnostic performance in the lung, and LNs located in cervical, mediastinum, supraclavicular, and mesenteric regions.

\section{Abbreviations}

ADC: Apparent diffusion coefficient; FLAIR: Fluid-attenuated inversion recovery; LN: Lymph node; PET/CT: Positron emission/computed tomography; T1-WI, T2-WI: T1- or T2-weighted images; WB-DWI-MRI: Wholebody diffusion-weighted magnetic resonance imaging

\section{Acknowledgements}

Not applicable.

\section{Authors' contributions}

$A A, E A, H I M$, and HGM conceived and designed the analysis. MK and HG collected the data. AT and HG performed the analysis, and HG wrote the manuscript. All authors read and approved the final manuscript.

\section{Funding}

This research received no specific grant from any funding agency in the public, commercial, or not-for-profit sectors.

\section{Availability of data and materials}

The datasets used and/or analyzed during the current study are available from the corresponding author on reasonable request.

\section{Ethics approval and consent to participate}

Ethics approval was obtained by Faculty of Medicine Ethical Committee, Assiut University, Egypt. Ethical approval was obtained in the meeting held by the committee on 11-05-2014. No reference number was issued by the committee. Signed committee approval is kept by the researchers. Written patients' informed consents for participation in this study were taken and kept.

\section{Consent for publication}

All patients included in this research gave written informed consent to publish the data contained within this study.

\section{Competing interests}

The authors declare that they have no competing interests.

\section{Author details}

${ }^{1}$ Radiology Department, Faculty of Medicine, Assiut University, Assiut, Egypt.

${ }^{2}$ Medical Oncology Department, Faculty of Medicine, Assiut University, Assiut,
Egypt. ${ }^{3}$ Surgery Department, Faculty of Medicine, Assiut University, Assiut, Egypt

Received: 17 April 2020 Accepted: 23 June 2020

Published online: 29 July 2020

\section{References}

1. Heindel W, Gubitz R, Vieth V, Weckesser M, Schober O, Schafers M (2014) The diagnostic imaging of bone metastases. Dtsch Arztebl Int 111(44):741-747

2. Kwee TC, van Ufford HM, Beek FJ, Takahara T, Uiterwaal CS, Bierings MB et al (2009) Whole-body MRI, including diffusion-weighted imaging, for the initial staging of malignant lymphoma: comparison to computed tomography. Invest Radiol 44(10):683-690

3. Pasoglou V, Michoux N, Larbi A, Van Nieuwenhove S, Lecouvet F (2018) Whole Body MRI and oncology: recent major advances. Br J Radiol 91(1090): 20170664

4. Albano D, Patti C, La Grutta L, Agnello F, Grassedonio E, Mule A et al (2016) Comparison between whole-body MRI with diffusion-weighted imaging and PET/CT in staging newly diagnosed FDG-avid lymphomas. Eur J Radiol 85(2):313-318

5. Balbo-Mussetto A, Cirillo S, Bruna R (2016) Whole-body MRI with diffusionweighted imaging: a valuable alternative to contrast-enhanced CT for initial staging of aggressive lymphoma. Clin Radiol 71(3):271-279

6. de Bondt RBJ, Nelemans PJ, Bakers F, Casselman JW, Peutz-Kootstra C Kremer B et al (2009) Morphological MRI criteria improve the detection of lymph node metastases in head and neck squamous cell carcinoma: multivariate logistic regression analysis of MRI features of cervical lymph nodes. Eur Radiol 19(3):626-633

7. Seber T, Caglar E, Uylar T, Karaman N, Aktas E, Aribas BK (2015) Diagnostic value of diffusion-weighted magnetic resonance imaging: differentiation of benign and malignant lymph nodes in different regions of the body. Clin Imaging 39(5):856-862

8. Sigovan M, Akl P, Mesmann C, Tronc F, Si-Mohamed S, Douek P et al (2018) Benign and malignant enlarged chest nodes staging by diffusion weighted MRI: an alternative to mediastinoscopy? Br J Radiol 91(1082):20160919

9. Massani M, Maccatrozzo P, Morana G, Fabris L, Ruffolo C, Bonariol L et al (2016) Diagnostic difficulties and therapeutic choices in intrapancreatic accessory spleen: case reports. Open Access Surgery 9:15-20

10. Sabri YY, Nossair EZB, Assal HH (2020) Role of diffusion weighted MRimaging in the evaluation of malignant mediastinal lesions. Egypt J Radiol Nucl Med 51:32

11. Jambor I, Kuisma A, Ramadan S (2016) Prospective evaluation of planar bone scintigraphy, SPECT, SPECT/CT, 18F-NaF PET/CT and whole body $1.5 \mathrm{~T}$ $\mathrm{MRI}$, including DWI, for the detection of bone metastases in high risk breast and prostate cancer patients: SKELETA clinical trial. Acta Oncol 55(1):59-67

12. Lecouvet FE, El Mouedden J, Collette L (2012) Can whole-body magnetic resonance imaging with diffusion-weighted imaging replace Tc $99 \mathrm{~m}$ bone scanning and computed tomography for single-step detection of metastases in patients with high-risk prostate cancer? Eur Urol 62(1):68-75

13. Balliu E, Boada M, Peláez I (2010) Comparative study of whole-body MRI and bone scintigraphy for the detection of bone metastases. Clin Radiol 65(12): 989-996

14. Stecco A, Trisoglio A, Soligo E, Berardo S, Sukhovei L, Carriero A (2018) Whole-Body MRI with Diffusion-Weighted Imaging in Bone Metastases: A Narrative Review. Diagnostics (Basel) 8(3):45

15. Koh D-M, Blackledge M, Padhani AR, Takahara T, Kwee TC, Leach MO et al (2012) Whole-Body Diffusion-Weighted MRI: Tips, Tricks, and Pitfalls. Am J Roentgenol 199(2):252-262

16. Padhani AR, Koh DM, Collins DJ (2011) Whole-body diffusion-weighted MR imaging in cancer: current status and research directions. Radiology 261(3): $700-718$

17. Duygulu G, Ovali GY, Calli C, Kitis O, Yunten N, Akalin T et al (2010) Intracerebral metastasis showing restricted diffusion: correlation with histopathologic findings. Eur J Radiol 74(1):117-120

18. Mehrabian H, Detsky J, Soliman H, Sahgal A, Stanisz GJ (2019) Advanced magnetic resonance imaging techniques in management of brain metastases. Front Oncol 9:440

19. Ahmed HAK, Mokhtar H (2020) The diagnostic value of MR spectroscopy versus DWI-MRI in therapeutic planning of suspicious multi-centric cerebral lesions. Egyptian Journal of Radiology and Nuclear Medicine 51(1) 
20. Berghoff AS, Spanberger T, Ilhan-Mutlu A, Magerle M, Hutterer M, Woehrer A et al (2013) Preoperative diffusion-weighted imaging of single brain metastases correlates with patient survival times. PLoS One 8(2):e55464

21. Lee CC, Wintermark M, Xu Z, Yen CP, Schlesinger D, Sheehan JP (2014) Application of diffusion-weighted magnetic resonance imaging to predict the intracranial metastatic tumor response to gamma knife radiosurgery. J Neuro-Oncol 118:351-361

22. Zakaria R, Das K, Radon M, Bhojak M, Rudland PR, Sluming V et al (2014) Diffusion-weighted MRI characteristics of the cerebral metastasis to brain boundary predicts patient outcomes. BMC Med Imaging 14:26

23. Paruthikunnan SM, Kadavigere R, Karegowda LH (2017) Accuracy of wholebody DWI for metastases screening in a diverse group of malignancies: comparison with conventional cross-sectional imaging and nuclear scintigraphy. Am J Roentgenol 209(3):477-490

24. Regier M, Schwarz D, Henes FO (2011) Diffusion-weighted MR-imaging for the detection of pulmonary nodules at 1.5 Tesla: intraindividual comparison with multidetector computed tomography. J Med Imaging Radiat Oncol 55(3):266-274

25. Liu J, Yang X, Li F, Wang X, Jiang X (2011) Preliminary study of whole-body diffusion-weighted imaging in detecting pulmonary metastatic lesions from clear cell renal cell carcinoma: comparison with CT. Acta Radiol 52(9):954-963

26. Tang J, Leung B, Lee D, Sung H, Cheng C (2019) Multimodality imaging spectrum of extranodal lymphoma: a pictorial review. Hong Kong J Radiol 22:67-77

27. Li B, Li Q, Chen C, Guan Y, Liu S (2014) A systematic review and metaanalysis of the accuracy of diffusion-weighted MRI in the detection of malignant pulmonary nodules and masses. Acad Radiol 21(1):21-29

28. Shenoy-Bhangle A, Baliyan V, Kordbacheh H, Guimaraes AR, Kambadakone A (2017) Diffusion weighted magnetic resonance imaging of liver: principles, clinical applications and recent updates. World J Hepatol 9(26):1081-1091

29. Riviere DM, van Geenen EJM, van der Kolk BM, Nagtegaal ID, Radema SA, van Laarhoven C et al (2019) Improving preoperative detection of synchronous liver metastases in pancreatic cancer with combined contrastenhanced and diffusion-weighted MRI. Abdom Radiol (NY) 44(5):1756-1765

30. Abou khadrah RS, Bedeer A (2019) A small hepatic nodule $(\leq 2 \mathrm{~cm})$ in cirrhotic liver: doTriphasic MRI and Diffusion-weighted image help in diagnosis. Egyptian Journal of Radiology and Nuclear Medicine 50(1).

\section{Publisher's Note}

Springer Nature remains neutral with regard to jurisdictional claims in published maps and institutional affiliations.

\section{Submit your manuscript to a SpringerOpen ${ }^{\circ}$ journal and benefit from:}

- Convenient online submission

- Rigorous peer review

- Open access: articles freely available online

- High visibility within the field

- Retaining the copyright to your article

Submit your next manuscript at $\boldsymbol{\nabla}$ springeropen.com 